Cuadernos de Lingüística Hispánica $n^{\circ} .24$

ISSN 0121-053X • ISSN en línea 2346-1829

Julio-Diciembre 2014, pp. 163-182

\title{
Minorías lingüísticas en Colombia. Acercamiento desde las políticas públicas y los derechos humanos*
}

DAVID LEONARDO GARCÍA LEÓN*

dgacr063@uottawa.ca

Recepción: 22 de febrero de 2014

Aprobación: 15 de abril de 2014

Cómo citar este artículo: García León, D. (2014). Minorías linguísticas en Colombia. Acercamiento desde las políticas públicas y los derechos humanos. Cuadernos de Lingüística Hispánica, 24, 163-182. Tunja: Uptc.

* Artículo de reflexión. Una versión preliminar fue presentada como ponencia en las II Jornadas de Jóvenes Lingüistas organizadas por la Universidad de Buenos Aires, Argentina.

* Estudiante becario del Doctorado en Español de la Universidad de Ottawa, Canadá. Magíster en Lingǘstica de la Universidad Nacional de Colombia y Licenciado en Humanidades español e inglés de la Universidad Pedagógica Nacional. Además es miembro del grupo de investigación Estudios del discurso de la Universidad Nacional de Colombia. 


\title{
Resumen
}

En el presente artículo se expone una aproximación a la situación de las minorías lingüísticas colombianas, desde una perspectiva de Políticas Públicas y Derechos Humanos. Para lograr este acercamiento, el artículo se estructura de la siguiente manera: se caracterizan los derechos lingüísticos y se registran los convenios internacionales que Colombia ha firmado en torno de este tema; se presentan las características más importantes de una perspectiva de políticas públicas basada en derechos humanos y se analiza, desde el enfoque mencionado, un documento de política pública que busca regular la situación de las lenguas minoritarias de Colombia, la Ley 1381 de 2010. Posterior a este análisis, se examina si los derechos lingüísticos están o no garantizados para las comunidades aquí estudiadas. Finalmente, se establece la necesidad de seguir el principio basado en los derechos humanos como perspectiva para mejorar la situación de las comunidades lingüísticamente minoritarias.

Palabras clave: derechos humanos, políticas públicas, políticas lingüísticas, minorías lingüísticas, educación bilingüe.

\section{Linguistic minorities in Colombia Analysis from Public policies and human rights}

\begin{abstract}
This article analyzes the condition of Colombian linguistic minorities, from the point of view of public policies and human rights. In doing so, this paper is structured as follows: linguistic rights are described and topic-related international agreements signed by Colombia are registered; the most important characteristics of human rights based public policies are presented, and a public policy document, seeking to regulate the state of Colombian minority languages (namely Law 1381 from 2010), is analyzed through the mentioned approach. Following this analysis, it is examined whether the communities' linguistic rights are guaranteed. Finally, the necessity of following the principles of human rights as an approach to improve the condition of minority linguistic communities is established.
\end{abstract}

Key words: human rights, public policies, linguistic policies, linguistic minorities, bilingual education.

164 


\section{Minorités linguistiques en Colombie Rapprochement des politiques publiques et les droits humains}

\section{Résumé}

Dans cet article, on expose une approximation à la situation des minorités linguistiques colombiennes, à partir d'une perspective de Politiques Publiques et de Droits Humains. Pour atteindre ce rapprochement, l'article est structuré ainsi: on caractérise les droits linguistiques et on signale les accords internationaux que la Colombie a signés, autour du sujet qui nous occupe. On présente les caractéristiques les plus importantes d'une perspective de politiques publiques basée sur les droits humaines. Et on analyse, à partir de l'approche citée, un document de politique publique qui cherche à régulariser la situation des langues minoritaires de la Colombie, la loi 1381 de 2010. Postérieur à cette analyse, on examine si les droits linguistiques sont ou non garantis pour les communautés étudiées ici. Finalement, on établit le besoin de poursuivre le principe basé sur le droits humains en tant que perspective pour améliore la situation des communautés linguistiquement minoritaires.

Mots clés: droits humains, politiques publiques, politiques linguistiques, minorités linguistiques, éducation bilingue.

\section{Minorias linguísticas na Colômbia Abordagem desde as políticas públicas e os direitos humanos}

\section{Resumo}

No presente artigo se expõe uma aproximação à situação das minorias linguísticas colombianas, desde uma perspectiva de Políticas Públicas e Direitos Humanos. Para lograr esta abordagem, 0 artigo se estrutura da seguinte maneira: se caracterizam os direitos linguísticos e se registram os convênios internacionais que a Colômbia tem assinado em torno deste tema; se apresentam as características mais importantes de uma perspectiva de políticas públicas baseada em direitos humanos e se analisa, desde o enfoque mencionado, um documento de política pública que busca regular a situação das línguas minoritárias da Colômbia, a Lei 1381 de 2010. Posterior a esta análise, se examina se os direitos linguísticos estão ou não garantidos para as comunidades aqui estudadas. Finalmente, se estabelece a necessidade de seguir o princípio baseado nos direitos humanos como perspectiva para melhorar a situação das comunidades linguisticamente minoritárias.

Palavras chave: direitos humanos, políticas públicas, políticas linguísticas, minorias linguísticas, educação bilíngue. 


\section{Introducción}

La convivencia de dos o más lenguas en un Estado es en la mayoría de los casos conflictiva, pues una de las lenguas se convierte en dominante y pone en riesgo la existencia de las otras como sucede en España con el vasco (Calvo Pérez, 2001) y en Haití con el criollo de base léxica francesa (Hebblethwaite, 2012). Este hecho conduce a que los Estados se vean en la obligación de regular la situación a través de la implementación de políticas lingüísticas. El caso colombiano es un claro ejemplo de esta situación, ya que en el país conviven oficialmente el español, dos lenguas criollas (el palenquero y el criollo sanandresano), la lengua Romaní y más de 60 lenguas indígenas. Este contacto es conflictivo en la medida en que la mayoría de las lenguas indígenas y criollas se encuentran amenazadas y en riesgo de desaparecer debido, entre otros factores, a la dominancia del español (Ardila, 2010; Sanmiguel, 2007). Este hecho ha conducido a que el Estado colombiano implemente políticas públicas con el fin de mejorar esta situación de conflicto; un claro ejemplo es la Ley 1381 de 2010, más conocida como la Ley de Lenguas, que busca, entre otros objetivos, preservar las lenguas minoritarias del país.

De esta manera, el presente artículo busca aproximarse a la situación de las minorías linguísticas colombianas desde una perspectiva de Políticas Públicas y Derechos Humanos. Esta perspectiva permite evidenciar la coherencia entre el discurso estatal y la realidad, pues su característica central es evaluar si existe una práctica real del derecho y no solo respaldo jurídico o legislativo

Para lograr el acercamiento mencionado, el artículo se estructura de la siguiente manera: en primer lugar, se caracterizan los derechos lingüísticos y se establecen los convenios internacionales que Colombia ha firmado en torno de este tema. Luego de esto, se presentan las características más importantes de una perspectiva de políticas públicas, basada en derechos humanos. En el tercer apartado, se analiza, desde el enfoque citado, un documento de políica pública que busca regular la situación de las lenguas minoritarias de Colombia, la ley 1381 de 2010. Posteriormente, se examina si los derechos lingüísticos están o no garantizados para las comunidades aquí estudiadas; en este apartado, se analizan

\section{6}


elementos como la educación y el problema del territorio, dado que estos están estrechamente vinculados con un ejercicio real de los derechos lingüísticos y humanos. Para terminar, el texto presenta algunas conclusiones e intenta establecer la necesidad de una perspectiva de derechos para mejorar la situación de las comunidades lingüísticamente minoritarias.

\section{Los derechos linguíísticos y sus implicaciones}

Los derechos lingüísticos forman parte de los derechos humanos fundamentales (Hamel, 1995) y se hacen valer a través de dos declaraciones internacionales: el convenio 169 de la OIT (Organización Internacional del Trabajo) y la declaración universal de los derechos lingüísticos promulgada por la UNESCO. La primera tiene como propósito un reconocimiento al principio de igualdad en relación con las diferencias colectivas e individuales; mientras que la declaración de la UNESCO establece la importancia del uso de la lengua materna en la escuela, reconoce el derecho a la promoción de las lenguas propias por parte de comunidades ancestrales y a usarlas en asuntos de diferente índole sociopolítico (Rojas-Primus, 2006). Algunos de los derechos más relevantes de esta declaración son los siguientes:

El derecho a la enseñanza de la propia lengua y cultura; el derecho a disponer de servicios culturales; el derecho a una presencia equitativa de la lengua y la cultura del grupo en los medios de comunicación; el derecho a ser atendidos en su lengua en los organismo oficiales y en las relaciones socioeconómicas.

(UNESCO, 1996).

Es importante destacar cuatro elementos centrales de la declaración de la UNESCO. Primero, el carácter individual y colectivo que se le da a la normativa. Para autores como Oriol (1997, p. 282) y Hamel (1993, p. 83), la declaración entiende que el ejercicio de los derechos lingüísticos solo se puede realizar en comunidad, pues es en el seno de esta en donde se usa la lengua. Este elemento es de suma importancia, en la medida en que los derechos lingüísticos son individuales y sociales al mismo tiempo, en oposición a los derechos fundamentales que se definen esencialmente como individuales (Hamel, 1995). Esto conduce a que en el plano individual, cada persona tenga el derecho a aprender y desarrollar su lengua materna y a usarla en contextos socialmente relevantes. En cuanto el plano colectivo, se entiende que las comunidades tienen derecho a mantener su identidad etnolingüística, a establecer sus propias escuelas y a mantener autonomía para administrar asuntos relacionados con la cultura y la lengua.

En segundo lugar, es importante destacar que la declaración abandona distinciones, tradicionalmente relevantes, que socavaban el uso de las lenguas en diferentes contextos. De esta manera, Oriol (1997) sostiene que: 
Minorías lingüísticas en Colombia. Acercamiento desde las políticas públicas y los derechos humanos

Uno de los esfuerzos de los redactores fue definir unos derechos lingüísticos equitativos, sin subordinarlos al estatus político o administrativo del territorio al que pertenece la comunidad lingüística, ni a criterios como el grado de codificación o el número de parlantes (p. 282).

Este hecho evidencia que las denominaciones como lenguas oficiales, nacionales, mayoritarias, modernas, entre otras, no tienen por qué afectar la garantía de los derechos proclamados en el documento. Este elemento está directamente vinculado con el problema del territorio, pues muchas naciones, entre ellas Colombia, han asignado el carácter de cooficialidad a las lenguas minoritarias, pero solo en los territorios donde originariamente viven estas comunidades. A la par, y siguiendo las ideas de este autor, la declaración "no contempla su reconocimiento como característica definitoria. Al mismo tiempo, no entiende el territorio tan solo como área geográfica, sino también como un espacio social y funcional imprescindible para el pleno desarrollo de la lengua" (Oriol, 1997, p. 286). De esta manera, los Estados no pueden excusar su responsabilidad por el hecho de haber establecido oficialidad territorial, pues el elemento esencial son los factores de uso de la lengua.

El compromiso por parte de los Estados de garantizar los derechos lingüísticos es uno de los elementos esenciales en la declaración. En esta, se sostiene que el Estado no puede excusarse en falta de recursos para el cabal cumplimiento de las obligaciones adquiridas (Oriol, 1997). Esto implica, como sostiene Lagerpetz (2001), que el Estado no puede ser neutral en cuanto a los fenómenos del mercado y debe tener una política de trato diferencial con las minorías lingüísticas, pues dado que sus lenguas no son en todos los casos elementos para acceder al mercado laboral, este factor puede contribuir a su desplazamiento. Además, sin subsidios públicos muchas escuelas bilingües no podrían sobrevivir dentro de la dinámica económica neoliberal actual (Rojas-Primus, 2006). Así, la garantía de los derechos linguísticos pasa por la asignación de los recursos necesarios y por una política de diferenciación positiva; elementos que permiten un goce efectivo de los derechos lingüísticos.

Otro elemento de suma importancia es la definición de asimilación que se da en la declaración. Esta es concebida como una decisión personal y libre de cualquier presión política, social o económica; es decir:

entendida como la aculturación de las personas en la sociedad que las acoge, de tal manera que substituyan sus características culturales de origen por las referencias, los valores y los comportamientos propios de la sociedad receptora -no debe ser en ningún caso forzada o inducida, sino el resultado de una opción plenamente libre- (UNESCO, 1996, p.20).

Este hecho evidencia que los Estados deben asumir una perspectiva de libertad de acción; esto significa que las circunstancias y condiciones de las minorías como el ambiente 
social y territorial, el género, la raza y la clase social no deben ser factores que conduzcan a la asimilación linguística y cultural, pues la decisión de asumir la cultura y la lengua del otro debe estar desligada de dichos factores y ser plenamente libre. Esto implica, siguiendo a Corredor (2010, p. 45), que el Estado debe ser garante de unas condiciones mínimas para que las personas puedan poner en acción sus capacidades y equiparar la desigualdad de oportunidades de las que son víctimas. Estos cuatro elementos llevan a considerar que la legislación internacional no solo busca una reivindicación y promoción de las lenguas minoritarias, sino que, en sus términos, busca "corregir los desequilibrios lingüísticos de manera que aseguren el respeto y el pleno despliegue de todas las lenguas y que establezcan los principios de una paz lingüística planetaria justa y equitativa, como factor principal de la convivencia social" (UNESCO, 1996, p. 24).

\section{Políticas públicas en perspectiva de derechos humanos}

En este apartado se establece qué es una política pública y qué implica su abordaje desde una perspectiva de derechos humanos. Para comenzar, las políticas públicas son mecanismos desarrollados por el Estado para regular, intervenir, proteger o promover un bien considerado como público y que genera conflicto (Roth, 2006). En este sentido, si se reconoce que una actividad genera injusticia, desigualdad u opresión, esta debe ser intervenida a través de la implementación de políticas públicas. Algunos autores definen las políticas públicas como:

Un conjunto conformado por uno o varios objetivos colectivos considerados necesarios o deseables, de medios y acciones que son tratados, por lo menos parcialmente, por una institución u organización gubernamental con la finalidad de orientar el comportamiento de actores individuales o colectivos para modificar una situación percibida como insatisfactoria o problemática (Roth, 2006, p. 66).

A la par, es importante tener en cuenta dos aspectos de las políticas públicas. El primero es que estas no se reducen a un cuerpo de normas. Las políticas públicas son el enlace entre el Estado, la sociedad y el ciudadano y, por consiguiente, son programas de acción que permiten la concreción de decisiones que reformen la sociedad (Roth, 2002). El otro aspecto para tener en cuenta es que son medios que buscan garantizar el cumplimiento y respeto de los derechos humanos. Esto significa que las políticas no son fines en sí mismos, sino herramientas para realizar los derechos humanos (Roth, 2006). De esta manera, tener a los derechos humanos como fines hace que las políticas públicas contribuyan a la emancipación individual y colectiva de los ciudadanos, pues, al garantizarlos, permite que sus acciones y decisiones sean llevadas a cabo en total libertad.

El último aspecto mencionado conduce a realizar una distinción entre el paradigma tradicional en políticas públicas y aquel que tiene un enfoque en derechos humanos. La 
primera diferencia se fundamenta en la selección del problema público para resolver. En el enfoque tradicional, se selecciona un problema específico y no se le vincula con la vulneración de un derecho. Por su parte, el enfoque aquí propuesto parte de establecer el problema desde la afectación de uno o más derechos (Vázquez \& Delaplace, 2011). Así, lo primero que se realiza, al momento de establecer la política pública, es identificar el quebrantamiento de un derecho y asumirlo como el problema público por resolver (Vázquez \& Delaplace, 2011). Un ejemplo de esto son los problemas educativos. El Estado puede identificar como problemática la falta de instalaciones educativas en una comunidad. Desde la perspectiva tradicional, se busca darle solución a través de políticas de construcción de infraestructura; mientras que, desde una perspectiva de derechos, se considera que este problema afecta el derecho a la educación y debe solucionarse con un enfoque más amplio; es decir, debe tener en cuenta aspectos como la accesibilidad a todas las personas sin discriminación alguna, la adaptabilidad a los contextos culturales y sociales requeridos y la aceptabilidad por parte de las comunidades en que se implementa.

Otra distinción relevante es en el modelo tradicional, para el Estado es importante hacer un uso racional de los recursos, al momento de implantar las políticas; es decir, el objetivo de este es contar con una gestión eficiente en términos de la inversión y no es claro qué actividades se dejan al mercado y cuáles deben ser reguladas por el Estado (Vázquez \& Delaplace, 2011). Por su parte, el enfoque de derechos enfatiza en las obligaciones que el Estado ha asumido al firmar declaraciones internacionales; significa que este se compromete a alcanzar dichos acuerdos a través de las políticas, aún si esto obliga, por ejemplo, a regular las prácticas del mercado, pues su fin son los derechos y no una gestión eficaz de los recursos. De esta manera, según Guendel (2002), se hacen necesarios dos elementos: "un respaldo jurídico en el cual se explicite el reconocimiento de los derechos específicos del grupo de personas que lo demandan y que se establezcan mecanismos pertinentes de exigibilidad y las regulaciones necesarias para garantizar la obligatoriedad de la ley” (p. 113).

Estas distinciones generan un cambio en cuanto a cómo se conciben los ciudadanos desde el Estado. Estos pasan de ser sujetos de necesidades a ser sujetos de derechos (Corredor, 2010). Este cambio es relevante, ya que una necesidad desaparece al ser satisfecha; sin embargo, los derechos permanecen y se convierten en el eje sobre el que se planea cualquier política. Esto permite que los alcances de las políticas sean de largo aliento y se vinculen con otras, generándose un marco de políticas garantes de derechos humanos. Esto no sucede en el enfoque tradicional, pues se solucionan necesidades que no se relacionan con problemas más estructurales y que con seguridad están presentes por la desprotección de uno o más derechos. El cambio de enfoque aquí expuesto también involucra las oportunidades y capacidades de los sujetos (Corredor, 2010). El Estado debe ampliar las oportunidades de las personas, garantizando sus bienes de mérito; es decir, aquellos derechos 
básicos que permiten el ejercicio de las capacidades individuales. Si las condiciones no están dadas para que el ciudadano pueda hacer uso de sus capacidades, es obligación del Estado modificar esta situación; esto hace que la desigualdad en el ejercicio de derechos desaparezca y que los ciudadanos puedan hacer uso de sus capacidades sin ninguna limitación.

Para finalizar la distinción de enfoques, es importante resaltar que la perspectiva de derechos empodera a los sujetos (Guendel, 2000). Esto sucede en la medida en que estos no son entendidos como beneficiaros de un programa, sino como participantes activos en la construcción de las políticas públicas. Así, se abandona la razón instrumental en donde el sujeto sufre los efectos de una política establecida por el Estado y se pasa a una emancipadora, cuyo fin es la construcción de una ciudadanía participativa que contribuya a una reforma social y cultural. Los ciudadanos están involucrados en todo el ciclo de vida de las políticas públicas: identificación del problema, formulación de la solución, toma de decisiones e implementación y evaluación- construyendo una cultura de la participación y generando cambios con todos los actores involucrados.

\section{La ley de lenguas, una mirada desde las políticas públicas y los derechos humanos}

La ley 1381 de 2010, más conocida como la ley de lenguas, surge como mecanismo legal para desarrollar la legislación ya existente en el país en cuanto las lenguas minoritarias. Este documento rescata los artículos 7, 8, 10 y 70 de la Constitución de 1991 y el convenio 169 de la OIT suscrito por Colombia. Es importante recordar que, en los artículos de la Constitución mencionados, se reconoce la diversidad étnica y cultural del país, se da carácter de cooficialidad a las lenguas de los grupos étnicos en sus territorios y se establece que para dichas comunidades la educación debe ser bilingüe. Basados en este marco legal, la ley de lenguas tiene como objetivo:

Garantizar el reconocimiento, la protección y el desarrollo de los derechos linguísticos, individuales y colectivos de los grupos étnicos con tradición lingüística propia, así como la promoción del uso y desarrollo de sus lenguas que se llamarán de aquí en adelante lenguas nativas" (Ley 1385, 2010).

Desde la perspectiva aquí propuesta, se podría afirmar que la ley tiene una tendencia garante de los derechos lingüísticos. En este documento, se establece que se debe garantizar el desarrollo de las lenguas de las minorías. Sin embargo, el rol del Estado en el documento es ambiguo, pues en algunos artículos, este tiende a la promoción mientras que, en otros, se deben garantizar los derechos educativos y linguísticos. Así, en el artículo 2 se establece que: «el Estado [...] promoverá la preservación, la salvaguarda y el fortalecimiento de las 
lenguas nativas, mediante la adopción, financiación y realización de programas específicos» (Ley 1385, 2010). Esto conduce a pensar que el papel del Estado no es de garantía, sino de promoción; este hecho puede afectar de manera negativa la realización de los derechos lingüísticos; pues como se estableció en el apartado anterior, el Estado tiene la responsabilidad de garantizar el ejercicio de los derechos y no puede asumir un rol pasivo en cuanto el goce efectivo del derecho, en este caso, el uso y mantenimiento de la lengua. La promoción de la preservación, salvaguarda y fortalecimiento, a través de diferentes programas no es suficientemente fuerte para mantener la vitalidad de las lenguas minoritarias, pues estas pueden desaparecer (Ardila, 2007, 2010; Moya, 2010) y, en realidad, requieren intervenciones directas que promuevan y permitan el uso de estos idiomas.

Por su parte, en el apartado referido a la educación, artículo 20, el papel del Estado pasa de la promoción a la garantía: "las autoridades educativas nacionales, departamentales, distritales y municipales y las de los pueblos y comunidades donde se hablen lenguas nativas, garantizarán que la enseñanza de estas sea obligatoria en las escuelas de dichas comunidades" (Ley 1385, 2010). Este punto pone sobre la mesa un hecho importante, el Estado se ve en la obligación de garantizar el derecho lingüístico a educarse en la lengua materna como lo consagra la declaración universal de los derechos lingüísticos promulgada por la UNESCO y como aparece en el artículo 10 de la Constitución del 91, en donde se asume una educación bilingüe para las comunidades con idiomas diferentes al español. De esta manera, al menos en el plano discursivo, se ve que existe una tendencia, -aunque algo ambigua- de las políticas públicas, al goce efectivo de los derechos lingüísticos plasmados en la legislación internacional.

Un aspecto importante por rescatar es: como política pública, la ley de lenguas ha asignado un rol importante a la participación de las comunidades involucradas. En el artículo 3, se establece un principio de concertación con las comunidades minoritarias. Esto significa que el Estado solo puede actuar bajo el reconocimiento y la sujeción de las decisiones de las comunidades. Este hecho reivindica el empoderamiento de los actores que participan en las políticas públicas, ya que desde una perspectiva de derechos humanos, la política pública debe contar con la participación de los actores y no puede ser de arribaabajo, es decir, no puede partir de una decisión unilateral del Estado, sino que, por el contrario, debe «adoptar el punto de vista de los actores a quienes incumbe el problema colectivo del que trata la política en cuestión, para luego interrogarse acerca de las reglas institucionales necesarias para resolverlo de una manera concreta y puntual» (Knoepfel, Larrue, Varone, \& Hinojosa, 2007, p. 26). Por lo tanto, hay, en la ley de lenguas, una tendencia de derecho a la autodeterminación que permite a las comunidades contar con un espacio de maniobra para tomar las decisiones que mejor consideren en cuanto el uso de sus lenguas.

\section{2}


Desde una mirada de derechos humanos, las políticas públicas no deben contener ningún tipo de discriminación (Vázquez \& Delaplace, 2011). Este hecho se evidencia en la Ley de lenguas, pues se sostiene que ningún hablante puede ser discriminado por el uso, transmisión o enseñanza de su lengua. A la par, este documento, en el artículo 5, enfatiza en el derecho al uso de los idiomas maternos de las comunidades lingüísticas minoritarias tanto en espacios públicos como privados:

Los hablantes de lengua nativa tendrán derecho a comunicarse entre sí en sus lenguas, sin restricciones en el ámbito público o privado, en todo el territorio nacional, en forma oral o escrita, en todas sus actividades sociales, económicas, políticas, culturales y religiosas, entre otras. (Ley 1385, 2010).

Este hecho es de suma importancia en la medida en que el territorio no se convierte en un factor de discriminación, ya que, como se evidenció en el apartado de los derechos lingüísticos, la legislación internacional da, a la funcionalidad de la lengua y no al territorio, el papel para garantizar los derechos. De esta manera, la Ley de lenguas mantiene la idea de que el territorio no puede limitar los derechos lingüísticos.

Es necesario tener en cuenta un aspecto más en el análisis de la Ley de lenguas: la ambigüedad en cuanto el enfoque asumido en el documento. No es posible saber con claridad si esta política pública parte de un enfoque de derechos o si busca solucionar un problema público especifico que no tiene relación con la vulneración de los derechos lingüísticos y educativos. Esto se debe a que, en todo el documento, no se menciona estos derechos ni como referentes para su promulgación ni dentro del cuerpo del documento. Esto hace pensar que la Ley parte de un enfoque tradicional en donde se busca dar solución a un problema específico, la desaparición de las lenguas minoritarias, sin relación con problemas más estructurales como la educación. Sin embargo, en los artículos 7, 8 y 9 sí se percibe los efectos negativos que podría traer el no respeto al uso de la lengua materna en relación con el acceso a derechos como la justicia y la salud. En la Ley se garantiza el uso de los idiomas minoritarios en el sistema de salud y de justica, previéndose que podrían vulnerarse estos derechos fundamentales si no se corrige el conflicto linguístico. Para cerrar este apartado, se puede sostener que la Ley de lenguas presenta dos características generales: coherencia en relación con la normativa internacional de derechos lingüísticos, y ambigüedad en cuanto al rol del Estado y al enfoque asumido. Estos dos aspectos pueden llevar a una separación entre la norma y la aplicación de la misma; es decir, entre el discurso y la práctica.

\section{Del discurso a la realidad}

En el acápite anterior, se realizó un breve análisis a un documento de política pública. Ahora es necesario evidenciar si el discurso propuesto desde el Estado es coherente con la realidad, pues como se mencionó en el apartado de políticas públicas, estas van más allá 
de la normatividad y abarcan los cambios que deben generarse en la realidad. También es importante mencionar que el marco normativo, en cuanto a las lenguas minoritarias, es mucho más amplio que el de la Constitución y el propuesto en la Ley de lenguas; documentos como la Ley General de Educación de 1994 también regulan la situación de estos idiomas, al establecer el tipo de educación para grupos étnicos, conocido como etnoeducación o educación bilingüe e intercultural.

A continuación, se analiza el estado de la educación para las comunidades lingüísticamente minoritarias, con el fin de determinar si se ha venido cumpliendo el objetivo de las diferentes políticas públicas en torno del tema; es decir, se tratará de evidenciar si el discurso corresponde con la realidad, al momento de hablar de políticas públicas para minorías lingüísticas.

Para comenzar, es importante recordar que uno de los derechos humanos básicos que poseen todas las personas es el derecho a la educación. Este derecho de subsistencia (Corredor, 2010) está directamente relacionado con los derechos lingüísticos, puesto que, en estos, se contempla el derecho a la enseñanza de la lengua materna y la cultura. Es relevante, entonces, tratar de identificar cómo se está ejerciendo el derecho a la educación en las minorías lingüísticas y ponerlo en relación con el tipo especial de educación que estas requieren: bilingüe, autodeterminativa e intercultural. Para ello, se toman los informes que el Programa de las Naciones Unidas para el Desarrollo (PNUD) generó en el 2011 en cuanto la situación de los indígenas y los afrocolombianos. En el caso de los afrocolombianos, nos centraremos en las poblaciones de San Basilio de Palenque, Cartagena y en el del Departamento de San Andrés, Providencia y Santa Catalina, debido a que, en estos dos territorios, es donde las dos lenguas criollas del país son habladas.

La perspectiva presentada en cuanto la situación de los indígenas por el PNUD no es alentadora. En el apartado sobre educación, el documento inicia afirmando que:

La situación de la educación para los indígenas colombianos tampoco es favorable, pues además de registrar altos índices de analfabetismo, acompañados de bajas tasas de escolaridad, tiene una cobertura restringida, se caracteriza por una participación marginal en el sistema educativo y enfrenta grandes vacíos de operatividad y funcionamiento (Pueblos indigenas. Diálogo entre culturas, 2012, p. 56)

Esto evidencia que el derecho a la educación se encuentra vulnerado. A la par, el hecho de que exista un alto índice de analfabetismo demuestra que existe una restricción al goce efectivo de los derechos lingüísticos. En estos, se establece que los grupos lingüísticamente minoritarios tienen el derecho a participar en la vida sociopolítica sin ninguna restricción lingüística. Si las comunidades minoritarias no manejan ningún código escrito, ni el de su lengua materna ni el de la comunidad dominante, es muy difícil que 
puedan ser ciudadanos con plenas capacidades en el Estado Colombiano, pues la cultura escrita es una característica esencial de las sociedades modernas y es necesario tener un dominio mínimo de la escritura para participar en ejercicios políticos básicos como el derecho al voto, por ejemplo. Este hecho, además, es resaltado por Lagerpetz cuando afirma:

Primero, la oportunidad de usar mi lengua materna, o cualquier otra lengua que domine, es esencial para mi vida cotidiana. Esta me vuelve capaz de comunicarme con mis vecinos, colegas y compañeros. Si no puedo hablar la lengua hablada por la gente que vive cerca de mí, o si la hablo muy imperfectamente, mi vida está destinada a ser difícil. Segundo, tengo que dominar la lengua usada en las administraciones, tanto públicas como privadas, que controlan la región en la que vivo. Sin conocer la lengua no puedo ni conocer mis deberes ni hacer valer mis derechos. Tercero, muchas funciones y profesiones están abiertas para mí sólo si tengo un dominio perfecto, o prácticamente perfecto, de la lengua local. Sin ese conocimiento, se me escaparán muchas oportunidades. Cuarto, puedo participar en actividades culturales, leer y escribir, escuchar y hablar, divertirme y educarme, sólo si conozco suficientemente bien la lengua usada en mi sociedad. Por último, en orden aunque no en importancia, puedo ser un miembro pleno de la polis democrática sólo si puedo participar en la discusión democrática (2001, p. 2).

De esta manera, el hecho de que exista un bajo índice de alfabetización en las comunidades indígenas no solo evidencia la vulneración al derecho a la educación y a los derechos lingüísticos, sino que, además, limita la actuación de estas comunidades como miembros plenos de una comunidad política. Cabe recordar que, desde una perspectiva de derechos, las políticas públicas no solo se centran en solucionar la afectación de un derecho, estas, además, deben contemplar cómo dicha afectación vulnera otros derechos (Vázquez \& Delaplace, 2011). En este orden de ideas, las políticas públicas para comunidades con códigos lingüísticos diferentes al español no solo no han garantizado el derecho a la educación, sino que, también, están quebrantando el derecho a la libre participación política.

En cuanto a las comunidades afrocolombianas que hablan lenguas minoritarias, la situación también es desfavorable. Según el informe del PNUD (Los Afrocolombianos frente a los Objetivos de Desarrollo del Milenio, 2012), los habitantes de San Basilio de Palenque presentan la tasa más elevada de analfabetismo y está muy alejada del promedio nacional (3,1\%) y mucho más de la meta propuesta por el ODM (Objetivos de Desarrollo del Milenio) de la ONU (Organización de las Naciones Unidas), como se evidencia en el gráfico 1. 


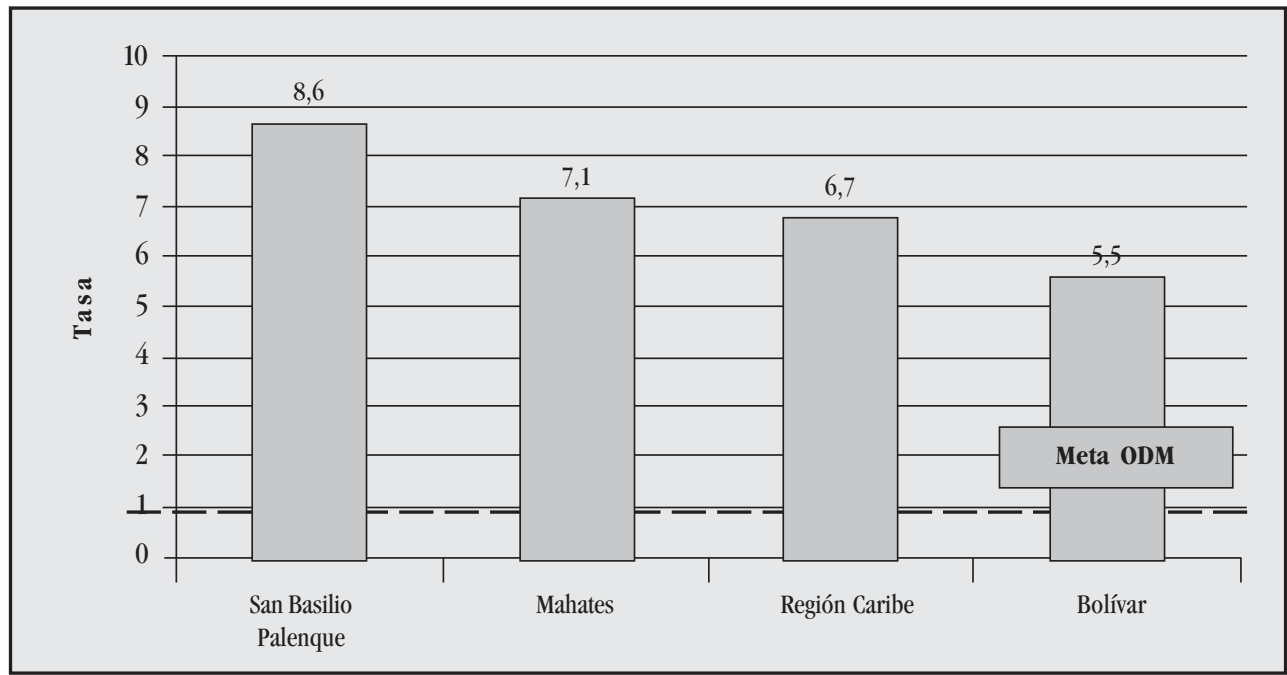

Tasas de analfabetismo en jóvenes de 15 a 24 años entre San Basilio de Palenque, el municipio, el departamento y la región a qué pertenecen.

(Los Afrocolombianos frente a los Objetivos de Desarrollo del Milenio, 2012, p. 70)

Además, los resultados en los exámenes de Estado ICFES ${ }^{1}$ para el 2008, también están por debajo del promedio departamental y municipal. Los estudiantes de Palenque obtienen un puntaje de 39,2 mientras que el promedio nacional está en 44,4 (Véase grafico 2). Esto evidencia que la calidad de la educación que recibe esta comunidad es muy inferior a la nacional. Además, es importante destacar que estos bajos resultados pueden deberse a una educación en lengua mayoritaria y no en la lengua materna de estos pobladores, el criollo palenquero. Es bien sabido por las investigaciones en educación bilinguie que se obtienen mejores resultados educativos cuando el proceso de alfabetización se da en la lengua materna y no se asume el proceso formativo totalmente en la segunda lengua (Baker, 1993, 2000). De esta manera, es posible afirmar que el derecho a una educación de calidad y acorde con las características lingüísticas de la población no está garantizado en esta comunidad. A la par, es importante evidenciar que, al igual que con los indígenas, otros derechos pueden estar vulnerados al no garantizarse, efectivamente, el derecho a la educación, pues como se plantea en el informe:

\footnotetext{
1 En Colombia, se evalúan los avances del aprendizaje frente a estándares básicos. Dichas evaluaciones incluyen el examen de Estado denominado prueba ICFES; este permite determinar las condiciones de alumnos de grado 11 al momento de ingresar a la educación superior.
}

\section{6}


El analfabetismo reproduce la pobreza por cuanto las ocupaciones remuneradas exigen como requisito tener por lo menos estudios primarios. De otra parte, constituye un impedimento serio para el cabal ejercicio de la ciudadanía, para participar con alguna posibilidad de éxito en sociedades del conocimiento, obtener y procesar información, tener voz, e intervenir conscientemente en el devenir de las comunidades a las que se pertenece (Los Afrocolombianos frente a los Objetivos de Desarrollo del Milenio, 2012, p. 57).

En cuanto San Andrés, el informe destaca un ligero avance; no obstante, esta comunidad sigue sufriendo los efectos de marginalización y racismo que caracteriza a toda la población afrocolombiana. Así, en relación con la tasa de analfabetismo, el archipiélago se encuentra en el nivel nacional, pero en cuanto la calidad de la educación que se mide en los resultados de las pruebas ICFES, el promedio es algo inferior (Los Afrocolombianos frente a los Objetivos de Desarrollo del Milenio, 2012, p. 81).

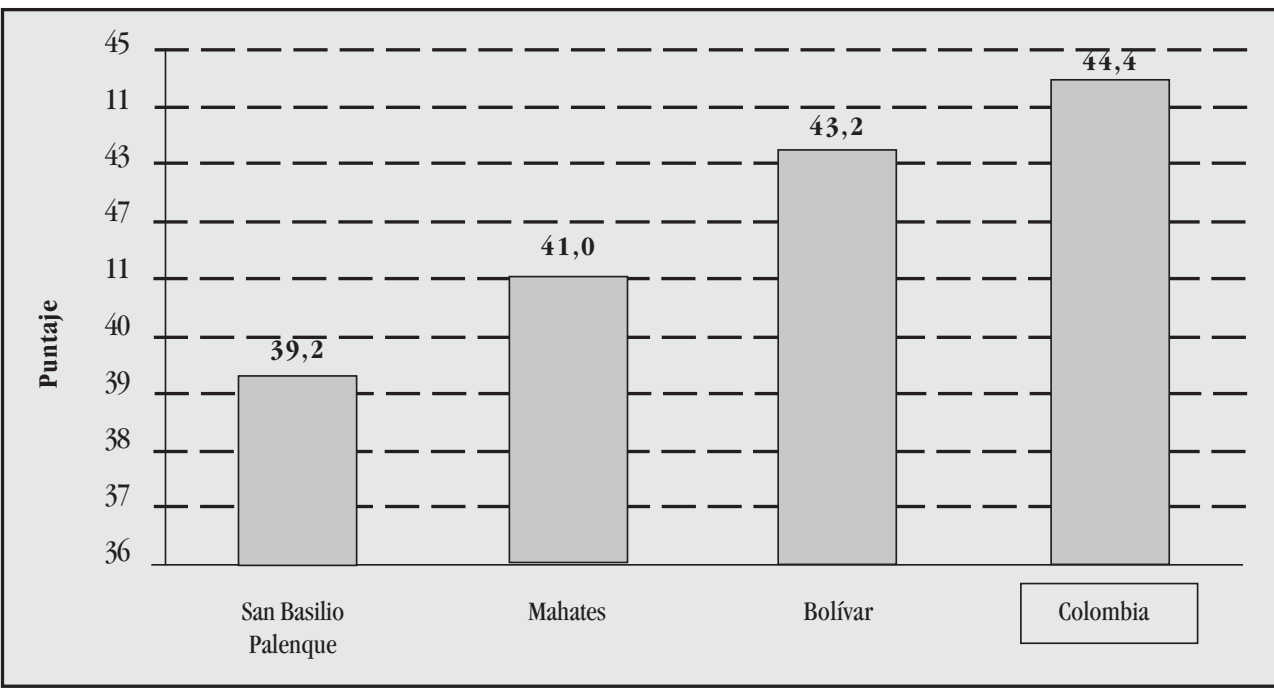

Gráfico 2

Puntajes promedio ICFES 2008 entre San Basilio de Palenque, el municipio y el departamento al que pertenecen.

(Los Afrocolombianos frente a los Objetivos de Desarrollo del Milenio, 2012, p. 83)

El problema de la educación va más allá, pues en lo relacionado con la etnoeducación, el informe destaca que esta se encuentra limitada por diferentes dificultades. En primer lugar, en la práctica no existe un trato diferencial para con estas comunidades (Pueblos 
indígenas. Diálogo entre culturas, 2012); esto genera la vulneración al derecho a tener una educación adecuada con las características lingüísticas y culturales propias de estos pueblos, consagrado en la declaración de la UNESCO y en la Ley de lenguas. Además, el informe destaca que el Estado no provee los recursos necesarios para que los programas de educación bilingüe se lleven a cabo, generando que, en la práctica, no haya un acceso real a este tipo de educación. Este fenómeno ha sido evidenciado por Montes quien sostiene:

La coyuntura reciente muestra -ante la etnoeducación- un Ministerio central que se limita a disposiciones formales y legalistas que han sido fuertemente criticadas, pues esas acciones han incidido adicionalmente en el debilitamiento del impulso etnoeducativo que requirió -y requeriría aún- la inyección de importantes recursos en investigación, capacitación y seguimiento. En los últimos tiempos, la fusión de instituciones y la eliminación de aquellas que no son viables por su bajo número de alumnos han afectado gravemente a las pequeñas escuelas indígenas dispersas en territorios de difícil acceso (2009, p. 132).

De esta manera, el Estado se excusa en la falta de recursos para no garantizar el derecho a una educación propia por parte de las comunidades minoritarias. Esto genera una vulneración directa a sus derechos y a los convenios firmados por Colombia. Otro punto por destacar son los currículos de la educación bilingüe: "no tienen en cuenta la identidad cultural y autonomía educativa de estos pueblos" (Los Afrocolombianos frente a los Objetivos de Desarrollo del Milenio, 2012, p. 58). Esto no solo afecta sus derechos lingüísticos, sino también su derecho a la construcción de una identidad propia, consagrada en el convenio 169 de la 0IT. Esto se debe, siguiendo a Soler (2011, p. 91), a una tendencia de homogenización y estandarización de la Educación en Colombia; los sistemas de evaluación por competencias propuestos en diferentes políticas educativas en la última década son contrarios a una educación diversa y conduce a la estandarización de la misma.

Todos los elementos aquí resaltados permiten afirmar que los derechos lingüísticos están consagrados, pero no garantizados. Además, el derecho a la educación propia se encuentra vulnerado. En resumen, y siguiendo a Castellanos y Cavides (2007, p. 267), las políticas públicas en torno de problemáticas lingüísticas y educativas no recogen las necesidades de los grupos minoritarios y convierten el derecho a la educación en un «espejismo», pues aunque pudiera servir para que dichas comunidades participaran en los procesos sociales, políticos y culturales propios y del país, la realidad muestra que esto no es posible, generando un estado no garantista de diferentes derechos, entre ellos los lingüísticos.

El último punto por tratar en este apartado es el problema del territorio en relación con los derechos lingüísticos. Como se mencionó en la sección de los derechos lingüísticos,

\section{8}


el territorio se entiende desde una perspectiva funcionalista de la lengua y no desde unos límites geográficos establecidos (Oriol, 1997). Además, se afirmó que este tipo de derechos son individuales y colectivos; es decir, necesitan de la existencia de una comunidad de habla para poder ser ejercidos (Hamel, 1993). Estos dos elementos conducen a pensar que un goce efectivo de los derechos lingüísticos se basa en la tenencia de un territorio propio en donde se asiente la comunidad lingüística; además, en cualquier situación de comunicación -independientemente del lugar- se debe garantizar el uso de la lengua materna. Estas dos consideraciones presentan dificultades en el contexto colombiano, dado que el conflicto armado ha generado altos índices de desplazamiento dentro de las comunidades indígenas y la situación de pobreza ha conducido a que estos migren a los municipios y a las grandes ciudades. El informe del PNUD sostiene que:

En los últimos años, la presencia de indígenas en las cabeceras de pequeños municipios y en las grandes ciudades ha ido creciendo como resultado del proceso migratorio de estos pueblos ocasionado por diferentes factores: cambios culturales y agotamiento de las tierras de los resguardos, en especial en la zona andina. Otro factor es el desplazamiento forzado de las comunidades a raíz de la lucha por la tierra de los resguardos por parte de actores armados ilegales (Pueblos indigenas. Diálogo entre culturas, 2012, p. 31).

Estos fenómenos generan que las comunidades no puedan usar sus lenguas nativas, lo cual contribuye a la pérdida de sus idiomas. Si a cada comunidad no se le garantiza un territorio común, no tendrá la posibilidad de construir una comunidad de habla en donde la lengua tenga utilidad. Además, la migración hacia regiones del país en donde hay presencia mayoritaria de hispanohablantes implica que los hablantes de lengua minoritarias no encuentren espacios de uso de sus lenguas y tengan que asumir el español como el único medio de comunicación. Por consiguiente, el problema del territorio y, en alguna medida, la pobreza, contribuyen a la negación de los derechos lingüísticos de las comunidades minoritarias del país. Estos dos problemas están, a la par, directamente vinculados con el desplazamiento ocasionado por el conflicto. Según destaca el informe sobre los pueblos indígenas, el desplazamiento de estas comunidades se ha incrementado, desde el 2005 al 2010 de manera desproporcionada, en comparación con el resto de la población; además, entre el 2002 y el 2010, 74.000 indígenas fueron desplazados (Pueblos indigenas. Diálogo entre culturas, 2012, p. 41). Por consiguiente, es necesario mejorar ciertas problemáticas estructurales en el país, para que las comunidades indígenas puedan gozar de sus derechos lingüísticos. Solo de esta manera, las políticas públicas generarán los efectos que se esperan y no tendrán solamente un valor discursivo. 
Minorías lingüísticas en Colombia. Acercamiento desde las políticas públicas y los derechos humanos

\section{Conclusión}

Las políticas públicas tienen como propósito dar solución a una situación de conflicto. Estas no son solo mecanismos legales, sino que son todas las herramientas que se ponen en acción. A la par, los problemas que estas buscan solucionar deben estar referidos a los derechos humanos, pues más que poner fin a unas necesidades, buscan garantizar unos derechos. De este modo, el presente texto evidenció que las políticas públicas colombianas en relación con los derechos lingüísticos se quedan en un plano discursivo y no pasan al goce efectivo de los mismos. El documento mostró cómo ciertas políticas lingüísticas son ambiguas en cuanto el papel del Estado y no asumen un enfoque de derechos. Además, se evidenció que no se garantizan los derechos lingüísticos y educativos de ciertas comunidades minoritarias. Todo esto reivindica la necesidad de que las políticas públicas del país comiencen a enfocarse en los derechos humanos. Esto permitiría una real aplicación de los convenios internacionales adquiridos. Adicionalmente, se espera que la perspectiva aquí asumida sirva como una herramienta para entender la situación sociolingǘstica y sociológica de las comunidades minoritarias y para analizar las políticas linguísticas del país. Por lo tanto, el derecho a una educación con calidad y de acuerdo con las necesidades linguísticas de las poblaciones con lenguas minoritarias en Colombia no está garantizado. Las políticas públicas no han cumplido con sus metas propuestas, pues se ratifican los derechos lingüísticos y humanos; sin embargo, el goce efectivo presenta bastantes deficiencias.

\section{Referencias bibliográficas}

Ardila, 0. (2007). Estudio sociolingüístico del Amazonas. Revista internacional Magisterio. Vol. 25, pp. 48-51.

Ardila, 0. (2010). Las lenguas indígenas de Colombia: problemáticas y perspectivas. UniverSOS Vol. 7, pp. 27-39.

Baker, C. (1993). Fundamentos de educación bilingüe y bilingüismo España: Cátedra.

Baker, C. (2000). A parents'and teachers' guide to bilingualism. USA: Multilingual Matters LTD.

Calvo Pérez, J. (2001). Contacto interlingüístico e intercultural en el mundo hispano. Valencia: Instituto Valenciano de Lengua y Cultura Amerindias.

Castellanos, M., \& Caviedes, M. (2007). La educación en los pueblos indígenas de Colombia. En: E. Rey (Ed.), Indigenas sin derechos. Situación de los derechos humanos de los pueblos indígenas. 2007 (pp. 265-280). Bogotá: Antropos.

180 
Corredor, C. (2010). La política social en clave de derechos. Bogotá: Universidad Nacional de Colombia.

Guendel, L. (2000). La política pública y la ciudadanía desde el enfoque de derechos humanos: la búsqueda de una nueva utopía. 170-218. Recuerado de Equidad para la infancia América Latina: http://www.equidadparalainfancia.org/la-politica-publica-y-laciudadania-desde-el-enfoque-de-los-derechos-humanos-la-busqueda-de-una-nuevautopia-580/index.html

Guendel, L. (2002). Políticas públicas y derechos Humanos. Revista de ciencias sociales. Vol. 3. No. 97. pp. 105-125.

Hamel, E. (1993). Derechos Lingüísticos. Revista Antropología. Vol. 13, No. 44, pp. 71-102.

Hamel, E. (1995). Derechos Lingüísticos como derechos humanos: debates y perspectivas. Alteridades. Vol. 5, No. 10, pp. 11-23.

Hebblethwaite, B. (2012). French and underdevelopment, Haitian Creole and development: Educational language policy problems and solutions in Haiti.Journal of Pidgin \& Creole Languages. Vol 27, No. 2, pp. 255-302.

Knoepfel, P., Larrue, C., Varone, F., \& Hinojosa, M. (2007). Hacia un modelo de análisis de políticas públicas operativo. Un enfoque basado en los acores, sus recursos y las instituciones. Ciencia Política. Vol. 3, pp. 6-29.

Lagerpetz, E. (2001). Sobre los derechos lingüísticos. Inonomía. Vol. 15, pp.109-130.

Ley 1385, 1385 C.F.R. (2010).

Los Afrocolombianos frente a los Objetivos de Desarrollo del Milenio (2012). Bogotá: Programa de las Naciones Unidas para el Desarrollo.

Montes, M. E. (2009). Tendencias actuales en la política linguística bilingüe en Colombia con menciones a la Amazonia. En: D. Aguirre (Ed.), Las lenguas autóctonas en Colombia. Consideraciones alrededor de su legitimación en la constitución de 1991. (pp. 117-141). Bogotá: Universidad de los Andes.

Moya, S. (2010). Situación sociolingüística de la lengua creole de San Andrés isla, el caso de San Luis. (Maestría en Lingüística), Universidad Nacional de Colombia, Bogotá.

Oriol, R. (1997). Declaración Universal de Derechos Linüísticos. Revista Iberoamericana de educación. Vol. 13, pp. 281-289.

Pueblos indigenas. Diálogo entre culturas. (2012). Bogotá: Progama de las Naciones Unidas para el Desarrollo.

Rojas-Primus, C. (2006). Diversidad lingüística y alfabetización en América Latina y el Caribe. Revista Iberoamericana de educación. Vol. 40, No. 3, pp. 1-11. 
Minorías lingüísticas en Colombia. Acercamiento desde las políticas públicas y los derechos humanos

Roth, A. (2002). Políticas públicas. Formulación, implementación y evaluación. Bogotá: Ediciones Aurora.

Roth, A. (2006). Discurso sin compromiso la política pública de derechos bumanos en Colombia. Bogotá: Aurora.

Sanmiguel, R. (2007). El bilingüismo en el Archipiélago de San Andrés, Providencia y Santa Catalina. Revista Internacional Magisterio. Vol. 25, pp. 28-31.

Soler, S. (2011). Análisis crítico del discurso de documentos de política pública en educación. Forma y Función. Vol. 24, No. 1, pp. 75-105.

Declaración universal de los derechos lingüísticos. (1996).

Vázquez, D., \& Delaplace, D. (2011). Políticas públicas con perspectiva de derechos humanos: un campo en construcción. Revista internacional de derechos humanos. Vol. 8, No. 14, pp. 35-65. 